J. Lake Sci. (湖泊科学) , 2013, 25(5):617-627

http: //www. jlakes. org. E-mail : jlakes@niglas.ac.cn

(C) 2013 by Journal of Lake Sciences

\title{
稳定同位素技术在地表水硝酸盐污染研究中的应用"
}

\author{
丁京涛 ${ }^{1}$, 席北斗 ${ }^{2 * *}$, 许其功 ${ }^{2}$, 高如泰 ${ }^{2}$, 卢 $义^{3}$, 黄 健 $^{1}$, 刘鸿亮 ${ }^{2}$ \\ ( 1 : 北京师范大学环境学院, 北京 100875 ) \\ (2: 中国环境科学研究院, 北京 100012) \\ ( 3 :重庆交通大学河海学院,重庆 400000$)$
}

\begin{abstract}
摘 要: 地表水硝酸盐污染已成为全球面临的主要水环境问题之一, 为了有效控制地表水中硝酸盐污染, 确定地表水中 硝酸盐的来源以及研究其在环境中的迁移转化过程就显得尤为重要. 硝酸盐稳定氮 $\left({ }^{15} \mathrm{~N}\right)$ 、氧 $\left({ }^{18} \mathrm{O}\right)$ 同位素作为一种有效 的示踪技术在识别地表水中硝酸盐的来源及迁移转化过程中已得到了广泛的应用, 但是硝酸盐在迁移转化过程中, ${ }^{15} \mathrm{~N} 、{ }^{18} \mathrm{O}$ 同位素会因为多种因素而发生分馏,同位素值发生变化使得这种技术的应用存在一定的局限性. 本文系统总结 了不同来源硝酸盐 $\delta^{15} \mathrm{~N} 、 \delta^{18} \mathrm{O}$ 值的组成特征及其影响因素, 分析了在不同土地利用类型流域内,利用硝酸盐 ${ }^{15} \mathrm{~N} 、{ }^{18} \mathrm{O}$ 同位 素技术开展硝酸盐来源识别、负荷估算和反硝化作用评估的方法和研究进展. 基于已有研究成果, 提出在未来的研 究过程中,一方面应以流域为单元,选择适当的研究方法并结合多种辅助指标研究多因素共同作用下 $\mathrm{NO}_{3}^{-}$污染源的 $\delta^{15} \mathrm{~N}^{-\mathrm{NO}_{3}^{-}}$和 $\delta^{18} \mathrm{O}-\mathrm{NO}_{3}^{-}$值的变化规律; 另一方面应对比流域污染源详细的调查数据, 分析现有负荷估算模型存在的不确 定性,并构建合适的负荷计算模型; 最后, 应深人开展同位素在确定流域和河流中反硝化发生的范围、地点和程度方面的 研究, 特别是掌握河流系统中沉积物一水体中氮的循环过程.
\end{abstract}

关键词: 硝酸盐; 污染源识别; 地表水; 稳定同位素; 反硝化

\section{Application of stable isotope on nitrate pollution researches of surface water}

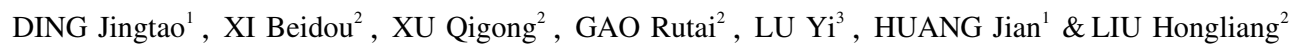 \\ (1: School of Environment, Beijing Normal University, Beijing 100875, P. R. China) \\ (2: Chinese Research Academy of Environmental Sciences, Beijing 100012, P. R. China) \\ (3: School of River and Ocean Engineering, Chongqing Jiaotong University, Chongqing 400000, P. R. China)
}

\begin{abstract}
Since nitrate contamination in surface water is an environmental problem in many regions of the world, methods to trace the source of nitrate and its transformation mechanisms are important for nitrate pollution control. Nowadays, stable nitrogen $\left({ }^{15} \mathrm{~N}\right)$ and oxygen $\left({ }^{18} \mathrm{O}\right)$ isotope data can provide meaningful insight for tracing sources of nitrate and have been frequently used to identify nitrate source and its transformation mechanisms in surface water. However, the stable isotope methods have limitations because the complex fractionations caused by multiple N-cycling processes can change the original isotopic values. This review paper systematically summarizes the isotopic composition and its influencing factors of various nitrate sources, the techniques and progresses in research on nitrate sources identification, and load estimation and assessment of denitrification in different types of land-use watersheds using nitrate stable isotope methods. Based upon the previous researches about the application of stable isotope methods, we suggest that in the future it is important to treat a catchment as a unit and to apply several assisted indexes to study the variation of $\delta^{15} \mathrm{~N}-N O_{3}^{-}$and $\delta^{18} \mathrm{O}-\mathrm{NO}_{3}^{-}$in different sources under the combined effects of multiple factors. In addition, comparative studies should be conducted to assess the uncertainty between polluting source survey data and the results of model simulation, and to build a better load computational model. Finally, further studies should be made to investigate the scale and extent of denitrification in a catchment/river using the nitrate stable isotope methods, especially in the sediment-water system.
\end{abstract}

Keywords: Nitrate; source identification; surface water; stable isotope; denitrification

* 国家水体污染控制与治理科技重大专项项目 (2012ZX07101-002) 资助. 2012-12-14 收稿; 2013-02-25 收修改 稿. 丁京涛,男,1984 年生, 博士研究生;E-mail:dingjingtao@ 163. com.

** 通信作者;E-mail: xibeidou@ 263.net. 
水体中硝酸盐 $\left(\mathrm{NO}_{3}^{-}\right)$污染可导致水体富营养化并严重威胁饮用水安全. 世界范围内的河流中接近 $70 \%$ 的氮是溶解性有机氮 ( DON), 而但其生物可利用度只有 $2 \% \sim 16 \%$, 硝酸盐是进人水环境中无机氮的主要成 分, 可被生物迅速利用 ${ }^{[1]}$. 尽管世界上许多地区实施了诸多的水污染控制措施, 如欧盟针对农业源硝酸盐污 染制订了详细的水体硝酸盐污染削减指令 ${ }^{[2]}$, 但水体中硝酸盐浓度仍然呈不断上升的趋势 ${ }^{[3]}$, 硝酸盐污染 已成为全球面临的主要水环境问题之一 ${ }^{[4-5]}$.

为了有效控制水体中硝酸盐污染, 识别其来源并估算不同源的污染负荷是实施各种污染控制措施的基 础工作. 目前通常采用统计学手段估算污染物从陆域迁移至水域的量 ${ }^{[6]}$. 但水体中硝酸盐与其它营养物质 类似, 其来源及迁移转化过程非常复杂, 不同源受到处理工艺、降水、地形、水文条件等诸多因素的影响, 尤 其是非点源污染具有的分散性、隐蔽性、随机性、不易监测和难以量化等特征 ${ }^{[7]}$, 使得 “陆域到水域” 的污染 物负荷统计学估算手段工作量大, 并存在很多不确定因素, 例如流域或水体中的反硝化作用发生的范围及 对硝酸盐的去除程度 ${ }^{[8]}$.

自从 1971 年 Kohl 等 ${ }^{[9]}$ 首次利用硝酸盐氮同位素 $\left({ }^{15} \mathrm{~N}\right)$ 评估农田化肥对河流中硝酸盐污染的影响以来, 稳定同位素作为一种有效的示踪技术在识别地表水中硝酸盐的来源及迁移转化过程中得到了广泛的应 用 $^{[10-13]}$. 虽然不同来源的硝酸盐往往具有不同的氮 $(N)$ 、氧 $(O)$ 同位素组成, 但是硝酸盐在迁移转化过程中, 影响同位素分馏的因素复杂, 其 $\mathrm{N} 、 \mathrm{O}$ 同位素值会因为许多物理、化学和生物作用而发生改变, 加之季节交替 引起的流域和水体温度及水文特征的变化, 使得稳定同位素技术在硝酸盐示源、硝化和反硝化过程研究中 存在许多不确定因素. 即便如此, 国外研究者开展了许多研究, 包括采样时间和采样方法的优化、将同位素 技术与流域水文资料、水质理化指标和土地利用数据等联合起来分析等多种手段, 以减少稳定同位素技术 在应用中的不确定性, 取得了较好的研究成果. 本文总结了地表水中硝酸盐 $\mathrm{N} 、 \mathrm{O}$ 稳定同位素技术在不同类 型流域地表水中硝酸盐来源的识别、硝酸盐在流域和河流中迁移转化特征等方面的研究方法及存在的问 题,并对该技术的发展进行了展望.

\section{1 硝酸盐 $N 、 O$ 同位素特征及其影响因素}

\section{1 不同来源硝酸盐 $N 、 O$ 同位素组成}

一般采用 $\delta$ 表示硝酸盐中 $\mathrm{N} 、 O$ 同位素的相对比值, 即样品的同位素比值相对于参照标准的同位素比值 的千分偏差(公式(1)). $\delta$ 为正值, 说明样品较参照标准富集同位素, 反之则说明样品较参照标准贫化同 位素.

$$
\delta_{\text {sample }}(\% 0)=\left[\left(R_{\text {sample }}-R_{\text {standard }}\right) / R_{\text {standard }}\right] \times 1000
$$

式中, $R$ 为同位素比值, 即元素的重同位素原子丰度与轻同位素原子丰度之比 $;{ }^{15} \mathrm{~N} 、{ }^{18} \mathrm{O}$ 的 $R$ 分别表示为 ${ }^{15} \mathrm{~N} /{ }^{14} \mathrm{~N} 、{ }^{18} \mathrm{O} /{ }^{16} \mathrm{O}$; standard 为参照标准. $\mathrm{N}$ 同位素参照标准是标准大气 $(\mathrm{AIR}), \mathrm{O}$ 同位素参照标准是维也纳标 准平均海水 (Vienna Standard Mean Oceanic Water, V-SMOW).

一般来说, 地球系统中的 $\delta^{15} \mathrm{~N}$ 值为 $-50 \% 0 \sim 100 \%$, 大多数含氮物质的 $\delta^{15} \mathrm{~N}$ 值集中于 $-10 \% 0 \sim 20 \% 0^{[14]}$. 土壤硝酸盐 $\delta^{15} \mathrm{~N}$ 值在 $0 \% 0 \sim 8 \%$ 之间 ${ }^{[15]}$; 大气中由于复杂的化学反应和不同 $\mathrm{N}$ 源的输人, 大气降水中 $\delta^{15} \mathrm{~N}$ 值浮动较大, 处于 $-13 \% 0 \sim 13 \%$ 之间 ${ }^{[16]}$; 大多数硝态氮肥是空气中氮气固定的产物, 其 $\delta^{15} \mathrm{~N}$ 值在 $-1 \% 0 \sim$ $2 \% 0$ 之间 ${ }^{[17-18]}$, 而有机肥具有更高的 $\delta^{15} \mathrm{~N}$ 值, 这是由于动物位于食物链较高级别, 加之其排泄物中的氮同位 素会在存储和处理过程中发生分馏作用, 如 $\mathrm{NH}_{3}$ 挥发会引起剩余的 $\mathrm{NH}_{4}^{+}$中富集 ${ }^{15} \mathrm{~N}$, 随着硝化反应的进行, 后续产生的 $\mathrm{NO}_{3}^{-}$中会逐渐富集 ${ }^{15} \mathrm{~N}^{[15]}$, 因此动物粪便产生的硝酸盐 $\delta^{15} \mathrm{~N}$ 值较高, 在 $8 \% \sim 22 \%$ 之间 ${ }^{[17]}$; 同 理, 人类生活污水也具有较高的 $\delta^{15} \mathrm{~N}$ 值, 可高于 $10 \%$, 若有工业来源或其它生活垃圾, 可能会低于 $10 \%{ }^{[19]}$, 而受生活污水影响的水生生态系统也明显富集 ${ }^{15} \mathrm{~N}^{[18]}$.

可见, 各种来源的硝酸盐 $\delta^{15} \mathrm{~N}$ 值存在重叠现象, 单纯使用 ${ }^{15} \mathrm{~N}$ 并不一定能够有效地识别硝酸盐来源, 而 硝酸盐 $\delta^{18} \mathrm{O}$ 值也是一种识别硝酸盐来源的指标 ${ }^{[20]}$, 可减少 $\mathrm{N}$ 同位素在识别硝酸盐来源时的不确定性 ${ }^{[21-22]}$. 降水中硝酸盐在形成过程中由于复杂的化学反应, 引起 ${ }^{18} \mathrm{O}$ 同位素分馏 ${ }^{[23]}$, 硝酸盐 $\delta^{18} \mathrm{O}$ 值范围较宽, 在 $25 \% 0 \sim 75 \%$ 之间, 较大气中的氧气 $\left(\delta^{18} \mathrm{O}\right.$ 为 $\left.23.5 \% 0\right)$ 富集 ${ }^{18} \mathrm{O}^{[18]}$; 硝态氮肥中的氧元素主要来源于氧气, 其 
$\delta^{18} 0$ 值分布于 $17 \% 0 \sim 25 \%$ 之间 ${ }^{[18]}$.

目前, 双同位素法 (dual isotope approach), 即同时分析 $\mathrm{NO}_{3}^{-}$中的 ${ }^{15} \mathrm{~N}$ 和 ${ }^{18} \mathrm{O}$ 同位素组成,已被作为河流、 海洋、湖泊水体和地下水中的 $\mathrm{NO}_{3}^{-}$来源识别的新技术手段在国外广泛应用 ${ }^{[15,24-25]}$.

\section{2 影响硝酸盐 N,O 同位素组成的因素}

自然界中含氮物质在迁移转化过程中 $N 、 O$ 同位素会因为一系列物理、化学和生物反应的影响而发生分 馏,使得水环境中的硝酸盐同位素组成并不能精确反映出来源的同位素组成. 影响硝酸盐同位素组成的因 素包括硝化作用、反硝化作用、固氮作用、同化作用、矿化作用、挥发、吸附和解析作用等.

1.2.1 硝化作用 硝化作用指铵盐在微生物作用下氧化为硝酸盐的过程. 微生物优先利用 ${ }^{14} \mathrm{~N}$, 使得剩余的 铵盐中富集 ${ }^{15} \mathrm{~N}$, 富集因子在 $-29 \%$ - $12 \%$ 。间 ${ }^{[16]}$, 随着硝化作用的进行, 后续产生的硝酸盐中会富集 ${ }^{15} \mathrm{~N}$. 在土壤中,一般认为硝化反应产生的硝酸盐中 $2 / 3$ 氧原子来自于水, $1 / 3$ 来自于 $\mathrm{O}_{2}$, 利用端元模型 (公式 (2) ) 可以计算出硝化反应产生的硝酸盐 $\delta^{18} \mathrm{O}$ 值的理论值,一般在 - 10\%o 10\%o之间,但自然条件下硝化反 应产生的硝酸盐 $\delta^{18} \mathrm{O}$ 值的实际值高于理论值 $5 \%$ 左右 ${ }^{[16,26-27]}$.

$$
\delta^{18}{\mathrm{O}-\mathrm{NO}_{3}^{-}}^{-}=2 / 3 \delta^{18} \mathrm{O}-\mathrm{H}_{2} \mathrm{O}+1 / 3 \delta^{18} \mathrm{O}-\mathrm{O}_{2}
$$

\section{2 .2 反硝化作用 影响硝酸盐同位素组成的} 另外一个因素是微生物的反硝化作用. 微生物 优先利用硝酸盐中轻同位素 ${ }^{14} \mathrm{~N} 、{ }^{16} \mathrm{O}$, 转化为 $\mathrm{N}_{2}$ 、 $\mathrm{N}_{2} \mathrm{O}$, 导致剩余的硝酸盐中富集重同位素 ${ }^{[12]}$, 反 硝化作用的 $\mathrm{N}$ 同位素富集因子一般在 $-40 \%$ $-5 \%$ 之间 ${ }^{[28]}, \mathrm{O}$ 同位素富集因子在 - 18\%o $-8 \%$ 之间 ${ }^{[21]}$. 反硝化引起的同位素分馏过程中, $\delta^{18} \mathrm{O}$ 与 $\delta^{15} \mathrm{~N}$ 比值在 $1: 1.3$ 到 1:2.1 之间 (图 $1)^{[13,15-16,29-31]}$. 有研究表明, 硝酸盐 $N 、 O$ 同位素组 成特征可以反映土壤、含水层、污水处理系统和 河岸带的反硝化作用, 但并不能明显反映水体中 沉积物厌氧层的反硝化作用导致的同位素分馏 效应 ${ }^{[21,28,32-33]}$.

1.2 .3 其它影响因素 氨挥发包括可逆反应( 溶 液中 $\mathrm{NH}_{3}$ 和 $\mathrm{NH}_{4}^{+}$之间、 $\mathrm{NH}_{3}$ 的气液相之间的挥 发) 和不可逆反应 (扩散损失), 都会导致 $\mathrm{NH}_{4}^{+}$富 集 ${ }^{15} \mathrm{~N}^{[15]}$; 若水体中硝酸盐浓度较高时, 浮游植物

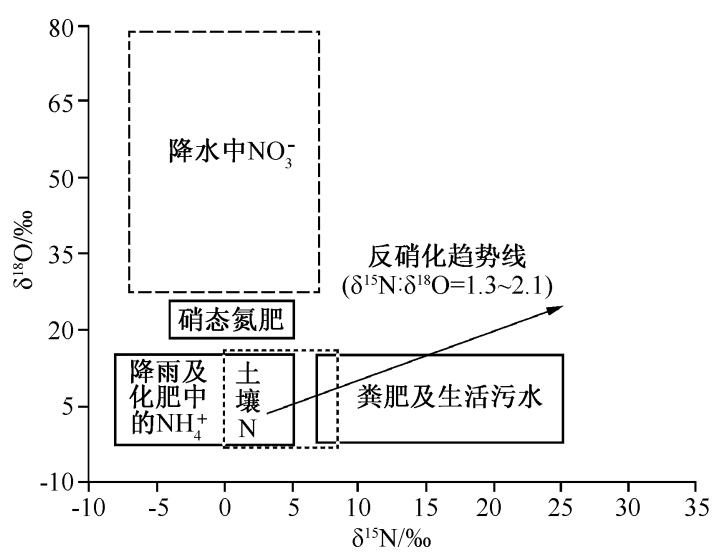

图 15 种硝酸盐源的 $\delta^{15} \mathrm{~N} 、 \delta^{18} \mathrm{O}$ 值 (据文献 $[4,15]$ 修改)

Fig. $1 \delta^{15} \mathrm{~N}$ and $\delta^{18} \mathrm{O}$ values of five potential nitrate sources ( modified from Nestler et $a l^{[4]}$ and Xue et $a l^{[15]}$ ) 的吸收可引起微弱的 ${ }^{15} \mathrm{~N}$ 同位素富集 ${ }^{[34]}$; 植物吸收利用铵盐、硝酸盐、亚硝酸盐的同化过程能引起较大范围 的氮同位素分馏, 范围在 $-27 \% 0 \sim 0 \%{ }^{[17]}$, 理论上, 同化吸收作用引起的 $\delta^{18} \mathrm{O} 、 \delta^{15} \mathrm{~N}$ 同位素分馏比率是 $1: 1^{[35]}$; 多种硝酸盐源的混合可改变水体硝酸盐 $\delta^{18} \mathrm{O}$ 和 $\delta^{15} \mathrm{~N}$ 值, 通常可根据质量平衡混合模型 (公式 (3)) 计算混合后的同位素值; 生物固氮作用是很多氮限制水体 (例如贫营养湖泊) 中氮素的重要来源 ${ }^{[36]}$, 虽然固 氮作用产生的含氮物质中的 $\delta^{15} \mathrm{~N}$ 值接近于大气 $\mathrm{N}_{2}$ 的 $\delta^{15} \mathrm{~N}$ 值 $(0 \%)^{[37-38]}$, 但固氮生物及其产生的有机物质 最终会矿化和/或经过硝化作用，从而间接影响硝酸盐的浓度及 $\delta^{15} \mathrm{~N}$ 值 ${ }^{[4,39]}$; 其它一些作用(矿化作用、吸附 解析过程等) 也可导致含氮物质发生很小的同位素分馏作用 ${ }^{[16]}$.

\section{2 不同类型流域地表水硝酸盐来源的同位素识别方法}

\section{1 土地利用类型较为单一的流域}

在以林地为主的流域, 土壤含氮有机物质硝化和降雨是硝酸盐的主要来源. 林地土壤自身的硝酸盐 $\delta^{15} \mathrm{~N}$ 值并没有土壤有机氮中的高 ${ }^{[40]}$, 而土壤中腐殖质富含 ${ }^{15} \mathrm{~N}$, 但一般被生物原位利用 ${ }^{[41]}$. 许多研究表明, 土壤 中的硝化反应是林地流域地表水中硝酸盐的主要来源 ${ }^{[23,42-43]}$. Johannsen 等 ${ }^{[4]}$ 利用公式 (2) 计算得到所研究 
河流水体中硝酸盐 $\delta^{18} \mathrm{O}$ 值为 $2 \% 0$, 与实测值 $(0.4 \% 0 \sim 2.3 \%$ ) 相近, 说明河流水体中硝酸盐主要来源于流域 土壤的硝化作用. Barnes 等 ${ }^{[45]}$ 分析美国东北部 5 个林地流域水体硝酸盐 $\mathrm{N} 、 \mathrm{O}$ 同位素发现,河流中 $82 \%$ $100 \%$ 的硝酸盐来源于土壤硝化反应. 水体硝酸盐浓度及同位素组成受温度 (影响微生物活性) 和降雨量 (影 响硝酸盐大气沉降) 的影响. 大气降水中大多数硝酸盐会被林地截留、植物吸收、发生迁移和转化 ${ }^{[23]}$, 使得 河流中硝酸盐 $\delta^{15} \mathrm{~N}$ 值比雨水中高 $5 \%$, 而降雨中硝酸盐由于同化吸收和硝化反应等原因, 使得 $\delta^{18} \mathrm{O}$ 值降 低 ${ }^{[45]}$. 当降雨量大时, 湿沉降会是一个重要的来源 ${ }^{[4]}$.

在以农业为主的流域, 水体中硝酸盐来源主要包括化肥及有机肥施用、大气沉降、 $\mathrm{N}_{2}$ 固定和土壤氮. 有 机肥和无机肥进人土壤后, 氮的轻同位素 ${ }^{14} \mathrm{~N}$ 较 ${ }^{15} \mathrm{~N}$ 优先发生氨 $\left(\mathrm{NH}_{3}\right)$ 挥发、植物吸收和反硝化作用, 从而使 得土壤中剩余的活性氮富集了 ${ }^{15} \mathrm{~N}$, 使得硝化反应成为土壤淋溶液中硝酸盐 $\delta^{15} \mathrm{~N}$ 明显升高的主要原因 ${ }^{[44,46]}$. 因此, 农田排水中硝酸盐的 $\delta{ }^{15} \mathrm{~N}$ 值并不能清楚地示源 ${ }^{[47]}$. 而当硝酸盐源在土壤中受微生物和矿化等作用影 响小, 并且硝酸盐能快速迁移出土壤时, 或者当土壤中微生物活性低时 (如冬天温度低的情况下), 在 $N 、 O$ 同位素的分馏作用受到一定限制时,双同位素法是一种有效的示源方法 ${ }^{[1,4]}$.

不同农作物和施肥方式影响着农田排水中硝酸盐浓度及 $\mathrm{N} 、 \mathrm{O}$ 同位素值. Kellman ${ }^{[48]}$ 研究了不同农作物 和施肥种类对农田暗管排水中硝酸盐 $\delta^{15} \mathrm{~N}$ 的影响, 发现施用无机肥的农田排水中硝酸盐的 $\delta^{15} \mathrm{~N}$ 值明显低 于施用有机肥 (猪粪) 的农田排水中的 $\delta^{15} \mathrm{~N}$, 农田排水中硝酸盐的 $\delta^{15} \mathrm{~N}$ 值普遍高于氮肥的 $\delta^{15} \mathrm{~N}$ 值, 说明存在 分馏效应. Deutsch 等 ${ }^{[1]}$ 发现农田暗管排水中硝酸盐的 $\delta^{15} \mathrm{~N} 、 \delta^{18} \mathrm{O}$ 值 $\left(\delta^{15} \mathrm{~N}=10.4 \%\right.$ 、 $\delta^{18} \mathrm{O}=4.7 \%$ 年地下水 中硝酸盐的同位素值 $\left(\delta^{15} \mathrm{~N}=0.6 \% 0 、 \delta^{18} \mathrm{O}=1.4 \% 0\right)$ 和降水中硝酸盐同位素值 $\left(\delta^{15} \mathrm{~N}=0.1 \% 0 、 \delta^{18} \mathrm{O}=51.7 \% 0\right)$ 有明显的区别, 当汇人河流后, 河水中硝酸盐同位素值 $\left(\delta^{15} \mathrm{~N}=9.0 \% 0, \delta^{18} \mathrm{O}=6.0 \% 0\right.$ ) 与农田暗管排水中的近 似,利用混合模型计算得出河流中有 $86 \%$ 的硝酸盐来自于农田暗管排水, 地下水和降雨分别占到 $11 \%$ 和 $3 \%$.

\section{2 受人类活动影响较大的混合流域}

在受人类生产生活影响较大的混合流域, 水体中硝酸盐主要来自生活和生产污水、化肥及有机肥施用、 土壤氮和大气沉降. 由于混合流域的污染源种类多, 影响同位素分馏的因素复杂. 国外研究者注重将同位素 技术与流域水文资料、水质理化指标和土地利用数据等联合起来分析流域及水体中硝酸盐污染的时空变化 特征.

2.2.1 与流域水文、降雨数据的联合分析 河流水文特征与降雨事件、季节变化和水力停留时间有关. 基于 降雨事件前后河流中硝酸盐的 $\delta^{18} \mathrm{O}$ 和 $\delta^{15} \mathrm{~N}$ 值, 可以有效识别降雨直接进人河流或通过地表径流进人河流 的硝酸盐负荷 ${ }^{[31,49]}$, 有时可以达到总负荷的 $67 \%{ }^{[50]}$; Kaushal 等 ${ }^{[13]}$ 利用质量平衡混合模型计算了降雨与废 水排放的硝酸盐负荷比例, 发现降雨事件中硝酸盐负荷比例值变动较大. 水文条件和温度的季节变化影响 着硝酸盐浓度及其同位素组成, Johannsen 等 ${ }^{[44]}$ 发现夏季河流中硝酸盐 $\delta^{15} \mathrm{~N} 、 \delta^{18} \mathrm{O}$ 值高于冬季, 而冬季水体 中硝酸盐浓度高于夏季, 这与夏季河流中微生物活性高和水生植物吸收利用强而引起的同位素分馏作用有 关系. Chen 等 ${ }^{[51]}$ 利用硝酸盐 $\delta^{15} \mathrm{~N} 、 \delta^{18} \mathrm{O}$ 值研究了太湖及其主要人湖河流中硝酸盐的来源, 发现夏季太湖水 体中硝酸盐主要来自大气沉降与生活污水/有机肥, 冬季北太湖主要来自污水和有机肥, 东太湖主要来自土 壤有机氮的硝化作用, 并指出硝化作用是太湖水体硝酸盐的重要来源. 同样, 水力停留时间可以影响微生物 和水生植物丰度, Kendall ${ }^{[16]}$ 指出当水体硝酸盐浓度较低、且微生物在水体中的滞留时间较短时, 同位素分馏 作用较小. 同样的结论得到了 Johannsen 等 ${ }^{[44]}$ 研究结果的证实.

2.2.2 与水质理化指标的联合分析 当硝酸盐来源多样时, 可以将硝酸盐 $\delta^{15} \mathrm{~N} 、 \delta^{18} \mathrm{O}$ 值与其它同位素值及水 质理化指标联合分析识别硝酸盐来源及源负荷. $\delta^{18} \mathrm{O}-\mathrm{H}_{2} \mathrm{O}$ 是一种分析不同类型流域河流在基流和暴雨事件 发生时降雨和水体硝酸盐变化特征的有效手段 ${ }^{[50]}$, 并且可用于辅助分析水体中硝酸盐的来源 ${ }^{[52]} \cdot \delta^{11} \mathrm{~B}$ 是区 分动物粪肥 (6.9\% 42.1\%o) 和化肥 ( $8 \%$ \% 17\% ) 的有效辅助指标 ${ }^{[15,53-54]}$. $\mathrm{Cl}^{-}$不易发生物理、化学和生物反 应, 是反映生活污水输人和稀释作用较好的指标 ${ }^{[55]}$. Liu 等 ${ }^{[55]}$ 、Koba 等 $^{[56]}$ 和 Chen 等 $^{[57]}$ 通过分析水体中 $\mathrm{NO}_{3}^{-} / \mathrm{Cl}^{-}$的摩尔比值可揭示水体的硝酸盐主要来自于化肥还是来自于动物粪肥和污水输人. Mayer 等 ${ }^{[12]}$ 指 出分析硝酸盐浓度与硝酸盐 $\delta^{15} \mathrm{~N}$ 值之间的关系, 可以得到水体 $\delta^{15} \mathrm{~N}$ 值升高的原因: 微生物反硝化作用可使 硝酸盐浓度减小而硝酸盐 $\delta^{15} \mathrm{~N}$ 值升高; 当两者值同时升高时, 可能是由于两个或多个硝酸盐来源导致的结 
果 (一个或一部分来源的硝酸盐浓度和 $\delta^{15} \mathrm{~N}$ 值低, 另一个或另一部分来源的硝酸盐浓度和 $\delta^{15} \mathrm{~N}$ 值高).

对于示踪水生系统硝酸盐各种氮的源和汇, 一个有用的助手是分析水体中颗粒态有机物 (POM) 的 $\delta^{15} \mathrm{~N}$ 和 $\delta^{13} \mathrm{C}$, 颗粒有机氮的 $\delta^{15} \mathrm{~N}$ 值在很大程度上取决于物源的 $\delta^{15} \mathrm{~N}$ 值, 研究 POM 氮同位素为揭示迁移转化过 程中氮素的利用提供了一种方法 ${ }^{[58]}$. POM 的 $\delta^{15} \mathrm{~N}$ 和 $\delta^{13} \mathrm{C}$ 反映了水体中颗粒态 $\mathrm{N} 、 \mathrm{C}$ 的各种来源以及有关的 生物地球化学过程 (例如光合作用、呼吸作用、反硝化作用等), 而这些过程又可改变溶解物的同位素 组成 ${ }^{[34]}$.

大多数河流特别是大型河流中 POM 主要是外源, 来源于流域侵蚀和水土流失过程; 而对于个别受人为 扰动较小的小型流域,有可能主要来自水体自身 (藻类 ${ }^{[34,59]}$. 河流中各种有机源的稳定同位素组成受到发 生在集水区内各种物理、生物地球化学和生物生理过程的影响,再加上环境条件、有机物来源类型和丰度等 方面的种种影响,综合反映为水系空间的和季节性的明显变化. Lehmann 等 ${ }^{[60]}$ 曾报道水体中 POM 的 $\delta^{15} \mathrm{~N}$ 值 的季节性变化可以反映其源的变化. Kendall 等 ${ }^{[61]}$ 对美国的四条大河 (密西西比河、科罗拉多河、格兰德河、 哥伦比亚河) 进行了碳氮稳定同位素分析, 认为浮游植物是河流颗粒有机碳的主要来源, 水库下游表现尤为 明显. 湖泊水体 POM 的 $\delta^{15} \mathrm{~N}$ 变化可以为湖泊内部营养元素被利用情况以及颗粒物向水体深部输移过程中 发生的相关生物地球化学过程提供重要信息,还能够为汇水区域内天然和人为活动保留较为详细和完整的 信息. Ostrom 等 ${ }^{[62]}$ 对 Superior 湖水体中的硝酸盐、颗粒态以及沉积有机物的元素组成及同位素值进行了测 定以推测它们的来源及循环转化过程,结果发现 $\delta^{15} \mathrm{~N}-\mathrm{POM}$ 主要受营养吸收与微生物降解过程引起的同位 素分馏的控制. Lehmann 等 ${ }^{[60]}$ 对 Lugano 湖颗粒物的 $\delta^{15} \mathrm{~N}$ 季节性变化进行了研究,试图验证稳定同位素技术 示踪富营养化湖泊内部生物地球化学过程以及有机物来源的有效性,并评估其保留高生产力湖泊生态系统 中初级生产力以及硝酸盐被利用情况的可靠性.

2.2 .3 与流域土地利用数据的联合分析 分析流域土地利用方式与水体中硝酸盐同位素之间关系可以更加 深人了解人类活动对硝酸盐排放的影响. Mayer 等 ${ }^{[12]}$ 分析了美国东北部 16 个流域/河流中硝酸盐 $\delta^{15} \mathrm{~N} 、 \delta^{18} \mathrm{O}$ 值, 得出河水 $\mathrm{NO}_{3}^{-}$中 $\delta^{15} \mathrm{~N}$ 与从废水量统计的 $\mathrm{N}$ 之间有显著的正相关关系 $\left(R^{2}=0.68, P<0.006, n=16\right)$, 还 求得此 $\delta^{15} \mathrm{~N}$ 与农业和城镇用地的比例存在显著正相关关系 $\left(R^{2}=0.75, P<0.001, n=16\right)$; Johannsen 等 ${ }^{[44]}$ 和 Voss 等 ${ }^{[63]}$ 也得到了相同的结论, 表明在以农业和城镇用地为主的流域, 河流中硝酸盐的高 $\delta^{15} \mathrm{~N}$ 值与生 活污水排放及有机肥施用有关.

\section{3 在大尺度的流域}

目前,利用双同位素法分析大尺度流域水体中硝酸盐的时空变化特征也得到了广泛应用. Battaglin 等 ${ }^{[64]}$ 利用质量平衡法和同位素法分析了密西西比河流域 (流域面积为 $2900000 \mathrm{~km}^{2}$ ) 的硝酸盐来源, 表明同 位素法对分析硝酸盐的来源、迁移转化机制及去除提供了有效的信息. Panno 等 ${ }^{[65]}$ 分析了伊利诺伊河 (Illinois River)（流域面积为 $78000 \mathrm{~km}^{2}$ ) 水体中硝酸盐 $\delta^{15} \mathrm{~N} 、 \delta^{18} \mathrm{O}$ 值, 揭示了水体中硝酸盐的来源及时间变化特 征: 丰水期硝酸盐主要来源是农田排水,生活污水处理设施对枯水期硝酸盐同位素影响较大. Li 等 ${ }^{[66]}$ 分析 了长江及其主要支流水体 (流域面积为 $1800000 \mathrm{~km}^{2}$ ) 中硝酸盐 $\delta^{15} \mathrm{~N}$ 值 $\left(7.3 \%\right.$ 12.9\%o) 和 $\delta^{18} \mathrm{O}$ 值 (2.4\%o 11.2\%o), 表明土壤有机氮的硝化作用和城镇污水排放是长江水体硝酸盐的主要来源; 水体中 $\delta^{15} \mathrm{~N}$ 和 $\delta^{18} O$ 值呈显著正相关 $\left(R^{2}=0.62\right)$,两者之比为 1.5 , 说明反硝化是重同位素富集的主要原因.

当然, 如果要详细分析大尺度流域内硝酸盐的污染源及污染特征, 还需要分析其它辅助指标, 如水文、 水质及土地利用数据等, 但利用硝酸盐双同位素法不失为一种有效而直接的方法.

\section{4 不同硝酸盐源负荷比例计算方法}

2.4.1 质量平衡混合模型 利用质量平衡混合模型 (a mass-balance mixing model) (公式 (3)) 确定硝酸盐源 负荷已得到了广泛的应用 ${ }^{[1,13,67]}$, 该方法要求硝酸盐源不能超过 3 个,且不同源的同位素值最好差异 较大 ${ }^{[67]}$.

$$
\begin{aligned}
& \delta^{15} \mathrm{~N}_{\text {MIX }}=f_{1} \delta^{15} \mathrm{~N}_{1}+f_{2} \delta^{15} \mathrm{~N}_{2}+f_{3} \delta^{15} \mathrm{~N}_{3} \\
& \delta^{18} \mathrm{O}_{\text {MIX }}=f_{1} \delta^{18} \mathrm{O}_{1}+f_{2} \delta^{18} \mathrm{O}_{2}+f_{3} \delta^{18} \mathrm{O}_{3} \\
& 1=f_{1}+f_{2}+f_{3}
\end{aligned}
$$


式中, $\delta^{15} \mathrm{~N}_{\mathrm{MIX}}$ 和 $\delta^{18} \mathrm{O}_{\mathrm{MIX}}$ 分别指混合样中 ${ }^{15} \mathrm{~N} 、{ }^{18} \mathrm{O}$ 的同位素比值, $1 、 2 、 3$ 代表 3 种来源, $f$ 代表不同源的硝酸盐 负荷比例 $(f \leqslant 3)$. Accoe 等 ${ }^{[54]}$ 将 $\delta^{11} \mathrm{~B}$ 也加人到公式 (3) 中, 可计算出 4 种源的负荷比例. 但是, 硝酸盐 $\delta^{15} \mathrm{~N}$ 、 $\delta^{18} \mathrm{O}$ 值的时空变化性、反硝化过程中同位素分馏作用和输人源较多等因素都可引起质量平衡混合模型计算 结果存在不确定性 ${ }^{[5,13,68-70]}$. 例如 $\delta^{15} \mathrm{~N} 、 \delta^{18} \mathrm{O}$ 值仅增加 $2 \%$, 可以导致地下水硝酸盐进入河流的负荷比例的计 算结果增加 $11 \% \sim 15 \%{ }^{[1]}$.

2.4.2 贝叶斯稳定同位素混合模型 为了减少质量平衡混合模型的不确定性, Parnell 等 ${ }^{[71]}$ 提出了贝叶斯稳 定同位素混合模型 (a Bayesian stable isotope mixing model) (公式 (4)), 将其集成在 R 统计软件中 (http:// cran. r-project. org/web/packages/siar/idex. html), 并综合分析了该模型与已在生态食物网研究上得到较好应 用的简单线性混合模型、IsoError、IsoSource、MixSIR 等模型的优缺点.

$$
X_{i j}=\sum_{k=1}^{K} P_{k}\left(S_{j k}+C_{j k}\right)+\varepsilon_{i j}, S_{j k} \sim N\left(\mu_{j k}, \omega_{j k}^{2}\right), C_{j k} \sim N\left(\lambda_{j k}, \tau_{j k}^{2}\right), \varepsilon_{i j} \sim N\left(0, \sigma_{j}^{2}\right)
$$

式中, $X_{i j}$ 为混合样 $i$ 中同位素 $j$ 的比值 $(i=1,2,3, \cdots, N ; j=1,2,3, \cdots, J) ; S_{j k}$ 为源 $k$ 中同位素 $j$ 的比值 $(k=1$, $2,3, \cdots, K$ ), 其平均值为 $\mu$, 标准偏差为 $\omega ; P_{k}$ 为所要计算的源 $k$ 的比例; $C_{j k}$ 为源 $k$ 中同位素 $j$ 的分馏因子 (平 均值 $\lambda$,标准偏差 $\tau$ ); $\varepsilon_{i j}$ 为混合样 $i$ 中同位素 $j$ 比值的残余误差 (平均值 0 ,标准偏差 $\sigma$ ).

目前, Xue 等 ${ }^{[5]}$ 利用 SIAR 模型计算得到了 3 种不同土地利用类型河流 5 种硝酸盐源 (降雨中硝酸盐、 化肥中硝酸盐、化肥和降雨中铵盐、土壤氮、有机肥和生活污水) 在冬季和夏季的贡献比例, 指出 SIAR 模型 计算结果不仅可以揭示硝酸盐的主要来源, 并且能同时揭示硝酸盐的其它重要来源, 可作为水体采样点的 硝酸盐源的 “指纹” 特征; 同时指出了 SIAR 模型的局限: 1) 地表水中硝酸盐 $\delta^{15} \mathrm{~N} 、 \delta^{18} \mathrm{O}$ 值的微小变化可能会 引起源负荷比例计算结果的较大变化;2) 硝酸盐的同位素组成会随着源输人呈现时间变化性, 加之不同源 的硝酸盐 $\delta^{15} \mathrm{~N} 、 \delta^{18} \mathrm{O}$ 值标准偏差较大, 使得 SIAR 模型计算出的源负荷比例范围较大.

\section{3 流域及水体中反硝化作用的同位素方法研究}

反硝化是氮循环的一个重要过程, 确定反硝化发生的范围和位置对地表水硝酸盐污染的控制和管理十 分重要. 研究表明, 双同位素法也可以更深人、详细地认识氮在传输过程中可能发生的生物地球化学过程, 有效地示踪湖泊和河流中的反硝化作用 ${ }^{[72-73]}$. 反硝化引起的同位素分馏过程中, $\delta^{18} \mathrm{O}$ 与 $\delta^{15} \mathrm{~N}$ 比值接近 $1: 2$, 但是, 水体中硝酸盐的同位素值的变化是流域过程和河流过程共同作用的结果, 反硝化作用引起的同位素 值的变化可能发生在河流迁移过程中,也可能发生在人河之前.

\section{1 表征流域反硝化程度}

大量研究表明, 硝酸盐在未进人河流之前, 流域反硝化作用是其消耗的重要原因, 并且通过 Rayleigh 方 程(公式(5)) 可以估算反硝化作用程度. Chen 等 ${ }^{[57]}$ 分析了亚热带河流北江河水体中 $4 、 8$ 和 12 月的硝酸盐 $\delta^{15} \mathrm{~N} 、 \delta^{18} \mathrm{O}$ 值, 发现水体硝酸盐主要来源于氮肥施用, 其次是生活污水和畜禽粪便, $4-8$ 月份水体中发生了 反硝化作用 $\left(\delta^{18} \mathrm{O}: \delta^{15} \mathrm{~N}=0.48\right)$, 并用 Rayleigh 方程计算了不同月份的反硝化程度,结果显示 4 月份水体同 位素值反映了反硝化作用较弱, 流域内硝酸盐的反硝化百分比为 $9.6 \%, 8$ 月份较为明显, 为 $48.1 \%$, 而 12 月份不明显, 可能是由于污水、有机肥污染的输人掩盖了反硝化作用的结果, 利用 Rayleigh 方程计算仍有 $25.8 \%$ 的硝酸盐发生了反硝化, 并指出水体季节之间的硝酸盐 $N 、 O$ 同位素关系并不能表明发生反硝化作用 的具体位置 (水体、土壤、地下水), 可以说是北江河流域内发生的反硝化作用在水体硝酸盐同位素值上的体 现. Panno 等 ${ }^{[8]}$ 的研究结果也表明, 密西西比河中硝酸盐 $O 、 N$ 同位素比值存在 $1: 2$ 的关系, 说明河流和流域 中的硝酸盐经历了明显的反硝化作用, 通过进一步分析发现, $0 \sim 55 \%$ 的硝酸盐在密西西比河上游流域内发 生了反硝化作用.

$$
\delta_{\mathrm{R}}=\delta_{\mathrm{R} 0}+\varepsilon \ln \left(S_{t} / S_{0}\right)
$$

式中, $\delta_{\mathrm{R}}$ 和 $\delta_{\mathrm{R} 0}$ 分别指反应物在反应进行 $t$ 时间和最初时的同位素值; $S_{t} / S_{0}$ 是指反硝化进行 $t$ 时间后反应物 浓度与初始浓度之比; $\varepsilon$ 是指同位素富集系数. 


\section{2 表征河流反硝化作用}

进人河流的氮元素中有相当一部分 $(37 \% \sim 72 \%$ ) 会由于河流过程 (生物吸收、沉积、渗漏到底部含水 层、水一沉积物界面的微生物反硝化作用) 而去除 ${ }^{[12,74]}$. 反硝化作用是从河流系统中永久去除氮的唯一途 径 ${ }^{[75]}$, 并且可直接并显著地改变硝酸盐氮同位素值 ${ }^{[76]}$. 除了反硝化作用导致河流硝酸盐浓度降低之外, 浓 度较低的硝酸盐的其他来源、水生植物的同化作用同样可以降低硝酸盐的浓度,使得反硝化作用在复杂环 境背景下的发生与否仅凭硝酸盐同位素和浓度的变化有时是很难判断的 ${ }^{[28]}$. Panno 等 ${ }^{[65]}$ 发现 Illinois 河在 低流量时,Peoria 湖 (与河流相连) 附近的水体中硝酸盐 $\delta^{18} \mathrm{O}$ 与 $\delta^{15} \mathrm{~N}$ 存在 $1: 2$ 的关系, 表明 Illinois 河经历着 很重要的反硝化作用,但同时指出 Illinois 河存在大量的硝酸盐损失 (在 2005 年夏天,每天损失 $7500 \mathrm{~kg}$, 约 占硝酸盐总负荷的一半), 这可能是反硝化作用和 (或) 生物吸收作用共同作用的结果. Kellman 等 ${ }^{[76]}$ 指出, 在小的 (流域面积为 $5 \mathrm{~km}^{2}$ )、施肥强度大的流域,水体反硝化的同位素特征表现得较明显. 而在大的河流(流 域) 内, 许多小而浅的支流增加了与河岸潜流带水力接触和物质交换的机会, 影响着 $\mathrm{N}$ 同位素值, 且随着河 流尺度的增大,这种水力接触和物质交换的机会就越大, $\mathrm{N}$ 损失越多,表征反硝化的同位素值变化越不 明显 ${ }^{[57,77]}$.

目前, 研究河流反硝化作用时, 一个有用的采样方法是拉格朗日采样法 (Lagrangian samping approach ${ }^{[4]}$, 有利于分析同一个水团中的营养物浓度及同位素值随时间的变化,进而分析河流中反硝化作用 程度. Battaglin 等 ${ }^{[64]}$ 通过对密西西比河下游进行拉格朗日采样并分析硝酸盐同位素值, 初步结果表明密西 西比河下游水体在春季或初夏期间氮损失的主要原因是藻类或其它水生植物的吸收,而不是反硝化作用. Deutsch 等 ${ }^{[75]}$ 利用拉格朗日采样法对德国易北河 (Elbe River) 7 月份水体进行为期 9 天的采样,发现硝酸盐 浓度随水流逐渐降低的同时, Chl. a 和 POC 浓度升高, 且 $\delta^{15} \mathrm{~N}: \delta^{18} \mathrm{O}$ 为 1 , 说明浮游植物的吸收对硝酸盐的吸 收是硝酸盐浓度降低的主要原因, 利用 $\mathrm{Cl}^{-}$质量平衡方法排除了地下水的影响,得出河流中硝酸盐负荷损失 量中有 $75 \%$ 是由于浮游植物的吸收作用, 最多有 $25 \%$ 的硝酸盐负荷损失是由河流反硝化作用引起的.

硝酸盐和铵氮的同位素值的变化可以有效地评估河流水体中反硝化作用 ${ }^{[78]}$,但也有研究指出硝酸盐 $\mathrm{N} 、 \mathrm{O}$ 同位素组成特征可以反映出土壤、含水层、污水处理系统和河岸带的反硝化作用,但并不能明显反映出 河流水体中沉积物厌氧层的反硝化作用导致的同位素分馏效应, 可能是因为充分混合的有氧水体中的硝酸 盐不断的向沉积物厌氧层扩散, 导致氮、氧同位素分馏效应不明显 ${ }^{[12,21,28,32-33]}$. 关于河流系统中沉积物一水体 中氮的循环过程, 还需要进一步研究 ${ }^{[44]}$.

\section{3 反硝化速率的确定}

在利用同位素有效地示踪反硝化作用的基础上,一些研究者开始探索利用同位素来定量示踪反硝化速 率. 主要是通过测量水体中硝酸盐和氮气中 ${ }^{15} \mathrm{~N} /{ }^{14} \mathrm{~N}$ 的自然丰度, 当反硝化作用是硝酸盐主要的去除机制 时, 由于反硝化引起较为明显的同位素分馏作用, 硝酸盐中 $\delta^{15} \mathrm{~N}^{-\mathrm{NO}_{3}^{-}}$值会有明显的增加, 所产生的氮气中 $\delta^{15} \mathrm{~N}$ 值会相应地降低 ${ }^{[79]}$. Ostrom 等 ${ }^{[80]}$ 在美国密歇根州一个小流域基于氮同位素分析数据建立水文模型来 定量反硝化速率, 研究了土壤一河流界面反硝化对硝酸盐的去除作用. Lehmann 等 ${ }^{[81]}$ 根据瑞士一个深水湖中 水体硝酸盐的氮、氧同位素剖面变化,利用简化的扩散反应模型估算了沉积物一水界面的水体硝酸盐氮、氧 同位素富集因子(分别为 $-20.7 \% 0 \pm 3.8 \%$ 和 $-11.0 \% 0 \pm 1.7 \%$ ) , 并探讨了反硝化速率与同位素富集因子的 关系. Sebilo 等 ${ }^{[78]}$ 利用硝酸盐、铵态氮浓度及 $\delta^{18} \mathrm{O}-\mathrm{NO}_{3}^{-} 、 \delta^{15} \mathrm{~N}^{-\mathrm{NO}_{3}^{-}} 、 \delta^{15} \mathrm{~N}-\mathrm{NH}_{4}^{+}$同位素技术得到法国塞纳河 河口反硝化速率约为 $0.02 \mathrm{mg} \mathrm{N} /(\mathrm{L} \cdot \mathrm{h})$. 研究者们认为, 同时分析硝酸盐中的氮、氧同位素是进行硝酸盐源 示踪并揭示其在水生生态系统中生物地球化学过程的有效办法 ${ }^{[81]}$, 为更好地理解同位素分馏与反硝化速率 的关系,还需要在更多及不同类型的地表水中开展研究.

\section{4 存在问题与展望}

目前,利用稳定氮氧同位素示踪技术进行硝酸盐污染示源及迁移转化研究中存在着较多的局限性和误 区, 如部分研究工作只是通过对比硝酸盐 $\delta^{15} \mathrm{~N}$ 值与不同来源 $\delta^{15} \mathrm{~N}$ 特征值的方法解析硝酸盐来源, 对硝酸盐 同位素值在迁移转化过程中的综合分析不够深人 ${ }^{[82-88]}$, 很可能对示源结果带来偏差. 但总体来说, 硝酸盐稳 定同位素技术对研究河流中硝酸盐来源、迁移、混合过程中同位素分馏特征和规律、研究硝酸盐在水体中的 
迁移转化特征、识别研究区域的硝酸盐来源以及弥补营养盐总量估算中遇到的困难和不足提供了实用的实 验手段和理论方法,具有广阔的应用前景.

1) 硝酸盐在迁移转化过程中稳定同位素值的变化. 硝酸盐氮循环各过程中复杂的同位素分馏可能改变 $\mathrm{NO}_{3}^{-}$污染源的 $\delta^{15} \mathrm{~N}^{-\mathrm{NO}_{3}^{-}}$和 $\delta^{18} \mathrm{O}-\mathrm{NO}_{3}^{-}$值, 使人们在利用 $\mathrm{N} 、 \mathrm{O}$ 稳定同位素判断 $\mathrm{NO}_{3}^{-}$污染源时产生偏差. 近 几年的研究表明, 以流域为单元, 把大气一植被一土壤一地表水一地下水作为一个系统, 采用适当的研究方法 (例如拉格朗日采样法), 结合现代信息技术和流域内的水文地球化学等指标 (例如 $\delta^{11} \mathrm{~B} 、 \mathrm{Cl}^{-} 、 \delta^{18} \mathrm{O}-\mathrm{H}_{2} \mathrm{O}$ 、 $\mathrm{NO}_{3}^{-} 、 \mathrm{NH}_{4}^{+}$浓度和 $\mathrm{POM}$ 等), 研究在混合作用、硝化、反硝化、生物利用等多种循环过程共同作用下 $\mathrm{NO}_{3}^{-}$污 染源 $\delta^{15} \mathrm{~N}-\mathrm{NO}_{3}^{-}$和 $\delta^{18} \mathrm{O}-\mathrm{NO}_{3}^{-}$值的变化规律, 有望在辨识硝酸盐污染的来源、定量估算不同源负荷及其迁移 转化机理等问题上取得突破.

2）硝酸盐源负荷比例计算模型及其不确定性分析. 虽然目前利用质量平衡混合模型确定硝酸盐源负荷 已得到了广泛的应用,但其计算结果存在较大的不确定性. 尤其是在较为复杂的混合流域,硝酸盐污染源种 类多样, 影响同位素分馏的因素众多,如何建立并优化水体中硝酸盐源负荷比例计算模型就成为一个重要问 题. 而 SIAR 模型是在质量平衡混合模型基础上的优化,但目前该模型应用较少, 对其计算结果的不确定分析 有待于提高. 另一个有用的方法是对比分析流域污染源调查数据及计算污染物总量平衡数据, 以此评估现有 计算方法在计算过程中存在的不确定性, 或开发其它计算模型, 弥补污染负荷统计估算常规方法的不足.

3) 流域、水体及沉积物中的反硝化作用对硝酸盐的去除过程. 反硝化是氮循环的一个重要过程, 双同位 素法可以更深人、详细地认识氮在传输过程中可能发生的生物地球化学过程, 但有研究表明, 硝酸盐 $N 、 O$ 同 位素组成特征可以反映土壤、含水层、污水处理系统和河岸带的反硝化作用, 但并不能明显反映水体中沉积物 厌氧层的反硝化作用导致的同位素分馏效应. 因此, 利用同位素技术研究流域和水体中反硝化发生的范围、地 点和程度, 掌握河流系统中沉积物一水体中氮的循环过程, 对掌握流域氮循环过程、有效控制和管理地表水硝 酸盐污染具有重要的意义. 关于河流系统中沉积物一水体中氮的循环过程,还需要进一步研究.

\section{5 参考文献}

[ 1 ] Deutsch B, Mewes M, Liskow I et al. Quantification of diffuse nitrate inputs into a small river system using stable isotopes of oxygen and nitrogen in nitrate. Organic Geochemistry, 2006, 37: 1333-1342.

[ 2 ] EC. Implementation of council directive 91/676/EEC concerning the protection of waters against pollution caused by nitrates from agricultural sources, 2002.

[ 3 ] 刘相超, 周政辉, 宋献方等. 梁滩河地表水与地下水水化学及硝酸盐污染. 重庆交通大学学报: 自然科学版,2009, 28 ( 5 ) :942-947.

[ 4 ] Nestler A, Berglund M, Accoe F et al. Isotopes for improved management of nitrate pollution in aqueous resources: review of surface water field studies. Environmental Science and Pollution Research, 2011, 18: 519-533.

[ 5 ] Xue DM, Baets BD, Cleemput OV et al. Use of Bayesian isotope mixing model to estimate proportional contributions of multiple nitrate sources in surface water. Environmental Pollution, 2012, 161: 43-49.

[ 6 ] 秦伯强,杨柳燕,陈非洲等. 湖泊富营养化发生机制与控制技术及其应用. 科学通报,2006,51:1857-1866.

[ 7 ] 贺缠生,傅伯杰,陈利顶. 非点源污染的管理及控制. 环境科学, 1998,19(5):87-91,96.

[ 8 ] Panno SV, Hackley KC, Kelly WR et al. Isotopic evidence of nitrate sources and denitrification in the Mississippi river, Illinois. Journal of Environmental Quality, 2006, 35(2) : 495-504.

[ 9 ] Kohl DH, Shearer GB. Fertilizer nitrogen: contribution to nitrate in surface water in a corn belt watershed. Science, 1971, 174: 1331-1334.

[10] Wells ER, Krothe NC. Seasonal fluctuation in $\delta^{15} \mathrm{~N}$ of groundwater nitrate in a mantled karst aquifer due to macropore transport of fertilizer-derived nitrate. Journal of Hydrology, 1989, 112 : 191-201.

[11] Feast NA, Hiscock KM, Dennis PF et al. Nitrogen isotope hydrochemistry and denitrification within the Chalk aquifer system of north Norfolk, UK. Journal of Hydrology, 1998, 211 : 233-252.

[12] Mayer B, Boyer EW, Goodale C et al. Sources of nitrate in rivers draining sixteen watersheds in the northeastern US: isotopic constraints. Biogeochemistry, 2002, 57/58: 171-197.

[13] Kaushal SS, Groffman PM, Band LE et al. Tracking nonpoint source nitrogen pollution in human-impacted watersheds. 
Environmental Science and Technology, 2011, 45(19) : 8225-8232.

[14] Freyer HD. Seasonal variation of ${ }^{15} \mathrm{~N} /{ }^{14} \mathrm{~N}$ ratios in atmospheric nitrate species. Tellus Series B-Hemical and Physical Meteorology, 1991, 43: 30-44.

[15] Xue DM, Botte J, Baets BD et al. Present limitations and future prospects of stable isotope methods for nitrate source identification in suface-and groundwater. Water Research, 2009, 43:1159-1170.

[16] Kendall C, McDonnell JJ. Isotope tracers in catchment hydrology. Amsterdam: Elsevier, 1998.

[17] Heaton THE. Isotopic studies of nitrogen pollution in the hydrosphere and atmosphere: a review. Chemical Geology, 1986, 59: 87-102.

[18 ] McClelland JW, Valiela I. Linking nitrogen in estuarine producers to land-derived sources. Limnology and Oceanography, 1998, 43 : 577-585.

[19] 周爱国,蔡鹤生,刘存富. 硝酸盐中 $\delta^{15} \mathrm{~N}$ 和 $\delta^{18} \mathrm{O}$ 的测试新技术及其在地下水氮污染防治研究中的进展. 地质科技 情报,2001,20(4) :94-98.

[20] Durka W, Schulze ED, Gebauer G et al. Effects of forest decline on uptake and leaching of deposited nitrate determined from ${ }^{15} \mathrm{~N}$ and ${ }^{18} \mathrm{O}$ measurements. Nature, 1994, 372: 765-767.

[21] Lehmann MF, Reicher TP, Bernasconi SM et al. Modeling nitrogen and oxygen isotope fractionation during denitrification in a lacustrine redox-transition zone. Geochimica et Cosmochimica Acta, 2003, 67 (14) : 2529-2542.

[22] Chang CCY, Kendall C, Silva SR et al. Nitrate stable isotope: tools for determining nitrate sources among different land uses in the Mississippi River Basin. Canadian Journal of Fisheries and Aquatic Sciences, 2002, 59: 1874-1885.

[23] Pardo LH, Kendall C, Pett-Ridge J et al. Evaluating the source of streamwater nitrate using $\delta^{15} \mathrm{~N}$ and $\delta^{18} \mathrm{O}$ in nitrate in two watersheds in New Hampshire, USA. Hydrological Processes, 2004, 18: 2699-2712.

[24] Seiler RL. Combined use of ${ }^{15} \mathrm{~N}$ and ${ }^{18} \mathrm{O}$ of nitrate and ${ }^{11} \mathrm{~B}$ to evaluate nitrate contamination in groundwater. Applied Geochemistry, 2005, 20 : 1626-1636.

[25] 陈法锦,李学辉,贾国东. 氮氧同位素在河流硝酸盐研究中的应用. 地球科学进展, 2007,22(12):1251-1257.

[26] Mayer B, Bollwerk SM, Mansfeldt T et al. The oxygen isotope composition of nitrate generated by nitrification in acid forest floors. Geochimica et Cosmochimica Acta, 2001, 65 : 2743-2756.

[27] Aravena R, Evans ML, Cherry JA. Stable isotopes of oxygen and nitrogen in source identification of nitrate from septic systems. Ground Water, 1993, 31: 180-186.

[28] Sebilo M, Billen G, Grably M et al. Isotopic composition of nitrate-nitrogen as a marker of riparian and benthic detrification at the scale of the whole Seine river system. Biogeochemistry, 2003, 63: 35-51.

[29] Böttcher J, Strebel O, Voerkelius S et al. Using isotope fractionation of nitrate-nitrogen and nitrate-oxygen for evaluation of microbial denitrification in a sandy aquifer. Journal of Hydrology, 1990, 114: $413-424$.

[30] Mengis M, Schiff SL, Harris M et al. Multiple geochemical and isotopic approaches for assessing ground water $\mathrm{NO}_{3}^{-}$elimination in a riparian zone. Ground Water, 1999, 37(3): 448-457.

[31] Burns DA, Boyer EW, Elliott EM et al. Sources and transformations of nitrate from streams draining varying land uses: evidence from dual isotope analysis. Journal of Environmental Quality, 2009, 38: 1149-1159.

[32] Brandes JA, Devol AH. Isotopic fractionation of oxygen and nitrogen in coastal marine sediments. Geochimica et Cosmochimica Acta, 1997, 61: 1793-1801.

[33] Reinhardt M, Müller B, Gächter R et al. Nitrogen removal in a small constructed wetland: An isotope mass balance approach. Environmental Science and Technology, 2006, 40: 3313-3319.

[34] Finlay JC, Sterner RW, Kumar S. Isotopic evidence for in-lake production of accumulating nitrate in Lake Superior. Ecological Applications, 2007, 17 (8) : 2323-2332.

[35] Granger J, Sigman DM, Needoba JA et al. Coupled nitrogen and oxygen isotope fractionation of nitrate during assimilation by cultures of marine phytoplankton. Limnology and Oceanography, 2004, 49(5) : 1763-1773.

[36] Mangaliso JG, Stephanie JG. Planktonic nitrogen fixation in Lake Malawi/Nyasa. Hydrobiologia, 2008, 596 (1): 251-267.

[37] Fry B. Stable isotope diagrams of fresh-water food webs. Ecology, 1991, 72: 2293-2297.

[38］王伟伟. 长江口潮滩营养动态与稳定同位素指示研究 [ 学位论文].上海:华东师范大学,2011.

[39] Michener R, Lajtha K. Stable isotopes in ecology and environmental science. British: Blackwell Publishing, 2007 : 183. 
[40] Spoelstra J, Schiff SL, Hazlett PW et al. The isotopic composition of nitrate produced from nitrification in a hardwood forest floor. Geochimica et Cosmochimica Acta, 2007, 71: 3757-3771.

[41] Billy C, Billen G, Sebilo M et al. Nitrogen isotopic composition of leached nitrate and soil organic matter as an indicator of denitrification in a sloping drained agricultural plot and adjacent uncultivated riparian buffer strips. Soil Biology and Biochemistry, 2010, 42: 108-117.

[42] Hales HC, Ross DS, Lini A. Isotopic signature of nitrate in two contrasting watersheds of Brush Brook, Vermont, USA. Biogeochemistry, 2007, 84: 51- 66.

[43] Piatek KB, Christopher SF, Mitchell MJ. Spatial and temporal dynamics of stream chemistry in a forested watershed. $H y$ drology and Earth System Sciences, 2009, 13: 423-439.

[44] Johannsen A, Dähnke K, Emeis K. Isotopic composition of nitrate in five German rivers discharging into the North Sea. Organic Geochemisty, 2008, 39: 1678-1689.

[45] Barnes RT, Raymond PA, Casciotti KL. Dual isotope analyses indicate efficient processing of atmospheric nitrate by forested watersheds in the northeastern U.S. . Biogeochemistry, 2008, 90 : 15-27.

[46] Wassenaar L. Evaluation of the origin and fate of nitrate in the Abbotsford aquifer using the isotopes of ${ }^{15} \mathrm{~N}$ and ${ }^{18} \mathrm{O}$ in $\mathrm{NO}_{3}^{-}$. Applied Geochemistry, 1995, 10: 391-405.

[47] Townsend-Small A, McCarthy MJ, Brandes JA et al. Stable isotopic composition of nitrate in Lake Taihu, China, and major inflow rivers. Hydrobiologia, 2007, 581 : 135-140.

[48 ] Kellman LM. A study of tile drain nitrate- $\delta{ }^{15} \mathrm{~N}$ values as a tool for assessing nitrate sources in an agricultural region. Nutrient Cycling in Agroecosystems, 2005, 71: 131-137.

[49] Anisfeld SC, Barnes RT, Altabet MA et al. Isotopic apportionment of atmospheric and sewage nitrogen sources in two connecticut rivers. Environmental Science and Technology, 2007, 41: 6363-6369.

[50] Buda AR, DeWalle DR. Dynamics of stream nitrate sources and flow pathways during stormflows on urban, forest and agricultural watersheds in central Pennsylvania, USA. Hydrological Processes, 2009, 23: 3292-3305.

[51] Chen ZX, Liu G, Liu WG et al. Identification of nitrate sources in Taihu Lake and its major inflow rivers in China, using $\delta^{15} \mathrm{~N}^{-\mathrm{NO}_{3}^{-}}$and $\delta^{18}{\mathrm{O}-\mathrm{NO}_{3}^{-}}^{-}$values. Water Science and Technology, 2012, 66 (3) : 536-542.

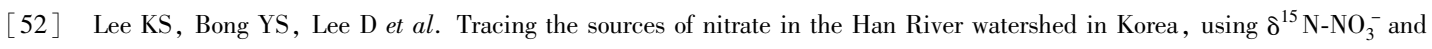
$\delta^{18} \mathrm{O}-\mathrm{NO}_{3}^{-}$values. Science of the Total Environment, 2008, 395: 117-124.

[53] Komor SC. Boron contents and isotopic compositions of hog manure, selected fertilizers, and water in Minnesota. Journal of Environmental Quality, 1997, 26: 1212-1222.

[54] Accoe F, Berglund M, Duta S et al. Source apportionment of nitrate pollution in surface water using stable isotopes of $\mathrm{N}$ and $\mathrm{O}$ in nitrate and B: a case study in Flanders (Belgium). European Commission, Joint Research Centre, Institute for Reference Materials and Measurements, Geel, EUR 23425 EN, 2008.

[55] Liu CQ, Li SL, Lang YC et al. Using delta N-15 and delta O-18 values to identify nitrate sources in Karst groundwater, Guiyang, Southwest China. Environmental Science and Technology, 2006, 40: 6928-6933.

[56] Koba K, Tokuchi N, Wada E et al. Intermittent denitrification: the application of a ${ }^{15} \mathrm{~N}$ natural abundance method to a forested ecosystem. Geochimica et Cosmochimica Acta, 1997, 61: 5043-5050.

[57] Chen FJ, Jia GD, Chen JY. Nitrate sources and watershed denitrification inferred from nitrate dual isotopes in the Beijiang River, south China. Biogeochemistry, 2009, 94: 163-174.

[58 ] Kumar S, Ramesh R, Bhosle NB et al. Natural isotopic composition of nitrogen in suspended particulate matter in the Bay of Bengal. Biogeosciences Discussions, 2004, 1: 87-105.

[59] 陶 贞, 高全洲, 姚冠荣等. 增江流域河流颗粒有机碳的来源、含量变化及输出通量. 环境科学学报, 2004,24 (5): 789-795.

[60] Lehmann MF, Bernasconi SM, McKenzie JA. Seasonal variation of the $\delta^{13} \mathrm{C}$ and $\delta^{15} \mathrm{~N}$ of particulate and dissolved carbon and nitrogen in Lake Lugano: Constraints on biogeochemical cycling in a eutrophic lake. Limnology and Oceanography, 2004,49 : 415- 429.

[61] Kendall C, Silva SR, Kelly VJ. Carbon and nitrogen isotopic compositions of Particulate organic matter in four large river systems across the United States. Hydrological Processes, 2001, 15(7) : 1301-1346. 
[62] Ostrom NE, Long DT, Bell EM et al. The origin and cycling of particulate and sedimentary organic matter and nitrate in Lake Superior. Chemical Geology, 1998, 152: 13-28.

[63] Voss M, Deutsch B, Elmgren R et al. Source identification of nitrate by means of isotopic tracers in the Baltic Sea catchments. Biogeosciences Discussions, 2006, 3: 663-676.

[64] Battaglin WA, Kendall C, Chang CCY et al. Chemical and isotopic evidence of nitrogen transformation in the Mississippi River, 1997-98. Hydrological Processes, 2001, 15: 1285-1300.

[65] Panno SV, Kelly WR, Hackley KC et al. Source and fate of nitrate in the Illinois River Basin, Illinois. Journal of Hydrology, 2008, 359(1/2) : 174-188

[66] Li SL, Liu CQ, Li J et al. Assement of the sources of nitrate in the Changjiang river, China using a nitrogen and oxygen isotopic approach. Environmental Science and Technology, 2010, 44: 1573-1578.

[67] Phillips DL, Koch PL. Incorporating concentration dependence in stable isotope mixing models. Oecologia, 2002, 130 : 114-125.

[68] Moore JW, Semmens BX. Incorporating uncertainty and prior information into stable isotope mixing models. Ecology Letters, $2008,11: 470-480$.

[69] Parnell AC, Inger R, Bearhop S et al. Source partioning using stable isotopes: coping with too much variation. PLoS One, $2010,5(3)$ : e9672.

[70] Phillips DL, Gregg JW. Source partitioning using stable isotopes: coping with too many sources. Oecologia, 2003, 136: 261-269.

[71] Parnell A, Inger R, Bearhop S et al. SIAR: Stable isotope analysis in R. , 2008.

[72] Middelburg JJ, Nieuwenhuize J. Nitrogen isotope tracing of dissolved inorganic nitrogen behavior in Tidal Estuaries. Estuarine, Coastal and Shelf Science, 2001, 53(3): 385-391.

[73] Harmelin-Vivien M, Loizeau V, Mellon C et al. Comparison of C and N stable isotope ratios between surface particulate organic matter and microphytoplankton in the Gulf of Lions (NW Mediterranean). Continental Shelf Research, 2008, 28 (15) : 1911-1919.

[74] Seitzinger PS, Styles RV, Boyer E et al. Nitrogen retention in rivers: model development and application to watersheds in the northeastern U.S. A. . Biogeochemistry, 2002, 57/58: 199-237.

[75] Deutsch B, Voss M, Fischer H. Nitrogen transformation processes in the Elbe River: Distinguishing between assimilation and denitrification by means of stable isotope ratios in nitrate. Aquatic Sciences, 2009, 71: 228-237.

[76] Kellman L, Marcel C. Nitrate cycling in streams: using natural abundance of $\mathrm{NO}_{3}^{-}-\delta^{15} \mathrm{~N}$ to measure in situ denitrification. Biogeochemistry, 1998, 43: 2273-2292.

[77] Peterson B, Wollheim W, Mulholland P et al. Control of nitrogen export from watersheds by headwater streams. Science, 2001, 292 : 86-90

[78 ] Sebilo M, Billen G, Mayer B et al. Assessing nitrification and denitrification in the Seine River and estuary using chemical and isotopic techniques. Ecosystems, 2006, 9: 564-577.

[79] Warneke S, Schipper LA, Bruesewitz DA et al. A comparison of different approaches for measuring denitrification rates in a nitrate removing bioreactor. Water Research, 2011, 45: 4141-4151.

[80] Ostrom NE, Hedin LO, VonFischer JC et al. Nitrogen transformations and $\mathrm{NO}_{3}^{-}$removal at a soil-stream interface: a stable isotope approach. Ecological Applications, 2002, 12(4) : 1027-1043.

[81] Lehmann MF, Sigman DM, Berelson WM. Couping the ${ }^{15} \mathrm{~N} /{ }^{14} \mathrm{~N}$ and ${ }^{18} \mathrm{O} /{ }^{16} \mathrm{O}$ of nitrate as a constraint on benthic nitrogen cycling. Marine Chemistry, 2004, 88: 1-20.

[82] 肖化云, 刘丛强, 李思亮等. 强水动力湖泊夏季分层期氮的生物地球化学循环初步研究: 以贵阳红枫湖南湖为例. 地球化学, 2002,6:571-576.

[83］肖化云,刘丛强.氮同位素示踪贵州红枫湖河流季节性氮污染. 地球与环境,2004,32(1):71-75.

[84] 陈能汪,洪华生,张珞平. 九龙江流域大气氮湿沉降研究. 环境科学, 2008,29(1):38- 46 .

[85] 陈惟财,陈伟琪,张珞平等. 九龙江流域地表水中硝酸盐来源辨析. 环境科学,2008,29(6):1484-1487.

[86] 王 珺, 高 高,裴元生等. 白洋淀府河中氮的来源与迁移转化研究. 环境科学,2010,31(12):2905-2910.

[87] 邢 萌,刘卫国,胡 婧. 浐河、涝河河水硝酸盐氮污染来源的氮同位素示踪. 环境科学, 2010,31(10):2305-2309.

[88］岳甫均,李 军,刘小龙等. 利用氮同位素技术探讨天津地表水氮污染. 生态学杂志,2010,29(7):1403-1408. 